\title{
High Expression of EphA4 Predicted Lesser Degree of Tumor Regression after Neoadjuvant Chemoradiotherapy in Rectal Cancer
}

\author{
Ching-Yih Lin ${ }^{1,2}$, Ying-En Lee ${ }^{3}$, Yu-Feng Tian ${ }^{4}$, , Ding-Ping Sun ${ }^{4}, 6$, Ming-Jen Sheu ${ }^{1}$, Chen-Yi Lin ${ }^{1}$, \\ Chien-Feng Li ${ }^{7}, 8,9,10$, Sung-Wei Lee ${ }^{11}$, Li-Ching Lin'12, I-Wei Chang13, Chieh-Tien Wang14,15, Hong-Lin \\ $\mathrm{He}^{13,16 \bowtie}$ \\ 1. Division of Gastroenterology and Hepatology, Department of Internal Medicine, Chi-Mei Medical Center, Tainan, Taiwan; \\ 2. Department of Leisure, Recreation, and Tourism Management, Southern Taiwan University of Science and Technology, Tainan, Taiwan; \\ 3. Department of Anesthesiology, Kaohsiung Chang Gung Memorial Hospital and Chang Gung University College of Medicine, Kaohsiung, Taiwan; \\ 4. Division of General Surgery, Department of Surgery, Chi Mei Medical Center, Tainan, Taiwan; \\ 5. Department of Health \& Nutrition, Chia Nan University of Pharmacy and Science, Tainan, Taiwan; \\ 6. Department of Pharmacy, Chia Nan University of Pharmacy and Science, Tainan, Taiwan; \\ 7. Department of Pathology, Chi Mei Medical Center, Tainan, Taiwan; \\ 8. National Institute of Cancer Research, National Health Research Institutes, Tainan, Taiwan; \\ 9. Department of Biotechnology, Southern Taiwan University of Science and Technology, Tainan, Taiwan; \\ 10. Institute of Clinical Medicine, Kaohsiung Medical University, Kaohsiung, Taiwan; \\ 11. Department of Radiation Oncology, Chi-Mei Medical Center, Liouying, Tainan, Taiwan; \\ 12. Department of Radiation Oncology, Chi-Mei Medical Center, Tainan, Taiwan; \\ 13. Department of Pathology, E-DA Hospital, I-Shou University, Kaohsiung, Taiwan; \\ 14. Department of Pathology, Chi Mei Medical Center, Liuying, Taiwan; \\ 15. Department of Medical Laboratory Science and Biotechnology, Chung Hwa University of Medical Technology; \\ 16. Institute of Biomedical Science, National Sun Yat-sen University, Kaohsiung, Taiwan.
}

$\triangle$ Corresponding author: Hong-Lin He, MD, Department of Pathology, E-DA Hospital, Kaohsiung, Taiwan. E-mail: baltic1023@gmail.com. Tel: +886-7-6150011 ext. 2903; Fax: +886-7-6150974

(C) Ivyspring International Publisher. This is an open access article distributed under the terms of the Creative Commons Attribution (CC BY-NC) license (https://creativecommons.org/licenses/by-nc/4.0/). See http://ivyspring.com/terms for full terms and conditions.

Received: 2016.09.05; Accepted: 2016.09.30; Published: 2017.04.09

\begin{abstract}
Background: Numerous transmembrane receptor tyrosine kinase pathways have been found to play an important role in tumor progression in some cancers. This study was aimed to evaluate the clinical impact of Eph receptor A4 (EphA4) in patients with rectal cancer treated with neoadjuvant concurrent chemoradiotherapy (CCRT) combined with mesorectal excision, with special emphasis on tumor regression.

Methods: Analysis of the publicly available expression profiling dataset of rectal cancer disclosed that EphA4 was the top-ranking, significantly upregulated, transmembrane receptor tyrosine kinase pathway-associated gene in the non-responders to CCRT, compared with the responders. Immunohistochemical study was conducted to assess the EphA4 expression in pre-treatment biopsy specimens from 172 rectal cancer patients without distant metastasis. The relationships between EphA4 expression and various clinicopathological factors or survival were statistically analyzed.

Results: EphA4 expression was significantly associated with vascular invasion $(P=0.015)$, post-treatment depth of tumor invasion $(P=0.006)$, pre-treatment and post-treatment lymph node metastasis $(P=0.004$ and $P=0.011$, respectively). More importantly, high EphA4 expression was significantly predictive for lesser degree of tumor regression after CCRT $(P=0.031)$. At univariate analysis, high EphA4 expression was a negative prognosticator for disease-specific survival $(P=0.0009)$ and metastasis-free survival $(P=0.0001)$. At multivariate analysis, high expression of EphA4 still served as an independent adverse prognostic factor for disease-specific survival (HR, 2.528; $95 \% \mathrm{Cl}, 1.131-5.651 ; P=0.024)$ and metastasis-free survival $(\mathrm{HR}, 3.908$; 95\% Cl, 1.590-9.601; $P=0.003)$.

Conclusion: High expression of EphA4 predicted lesser degree of tumor regression after CCRT and served as an independent negative prognostic factor in patients with rectal cancer.
\end{abstract}

Key words: EphA4, rectal cancer, chemoradiotherapy, CCRT. 


\section{Introduction}

With the advance in economic development and the change in dietary habit, the incidence of colorectal cancer has been rising dramatically in recent two decades. Currently, colorectal cancer is the third leading cause of cancer deaths in both males and females in Taiwan [1]. Surgery is the main treatment for early rectal cancer that has not spread to distant organs. For locally advanced rectal cancer, preoperative concurrent chemoradiotherapy (CCRT) followed by surgical resection is the first choice. Neoadjuvant CCRT can result in cancer shrinkage and make surgery more effective for larger tumors. It also lowers the rate of local recurrence [2-4]. Hence, it is of great importance to search for potential biomarkers that can predict the CCRT response. It can provide information for risk stratification, and additional treatment strategies may be applied for rectal cancer patients with poor response to current treatment. The improvement in bioinformatic tools to analyze copious gene expression microarray data has shed light on identifying potential biomarkers to predict the response of treatment.

Numerous receptor tyrosine kinases have been found to play an important role in tumor progression in a wide variety of cancers. Initially, we analyzed the public expression profiling dataset (GEO: GSE35452) of rectal cancer receiving neoadjuvant CCRT [5]. We particularly focused on genes associated with transmembrane receptor protein tyrosine kinase signaling pathway, and found that Eph receptors A4 $(E p h A 4)$ was the first significantly upregulated gene in the non-responders to neoadjuvant CCRT, compared with the responders. Therefore, we focused on EphA4 as a molecular target for further validation in a large cohort of patients with rectal cancer. The Eph receptors belong to the subfamily of transmembrane receptor tyrosine kinases. They are activated by a group of cell surface-anchored ligands, called ephrins. To date, $16 \mathrm{Eph}$ receptors have been identified. They are divided into two subclasses, A and B based on their structures and binding affinities for the ephrin ligands [6]. These Eph receptors and their ligands are involved in a number of biologic functions, including neural development, neovascularization, tumorigenesis and metastasis [7-9]. Many studies have correlated Eph and ephrin expression levels with cancer progression, metastasis and patient survival. EphA4, for example, was upregulated in glioma, gastric cancer, pancreatic cancer, and colorectal cancer [10-13]. However, little is known about the correlation between EphA4 expression and the response of CCRT in rectal cancer.

In this study, we assessed the protein expression levels of the EphA4 in 172 pairs of cancer tissue and adjacent normal mucosa obtained from patients with rectal cancer receiving neoadjuvant CCRT followed by surgery. We tried to clarify the relationships between the expression of EphA4 and various clinicopathological characteristics, particularly tumor regression after CCRT. To assess the prognostic significance, the association of EphA4 expression with survival was analyzed.

\section{Materials and Methods}

\section{Data mining of gene expression profiles of rectal cancer}

We tried to analyze the publicly available gene expression microarray dataset of rectal cancer (accession number: GSE35452) in Gene Expression Omnibus database [5]. This dataset included 46 rectal cancer patients treated with neoadjuvant radiotherapy combined with mesorectal excision. We focused on genes associated with transmembrane receptor protein tyrosine kinase signaling pathway and differentially expressed between responders and non-responders to neoadjuvant therapy. We analyzed the raw Affymetrix image files (CEL) of this dataset with the software Nexus Expression 3 (BioDiscovery, EI Segundo, California, USA). Analytic genes that meet the criteria of $\log 2$ fold change more than \pm 0.1 and $P$ value less than 0.01 were selected for further analysis.

\section{Patients and tissue samples}

The study group consisted of 172 patients with primary rectal adenocarcinomas who underwent neoadjuvant CCRT followed by mesorectal excision from January 1998 through December 2004 at Chi Mei Medical Center (Tainan, Taiwan). Those with distant metastasis and/or positive resection margin (includes circumferential radial margin) were excluded. This study was approved by the Institutional Review Board of Chi Mei Medical Center (IRB10501-008). The tumor tissues for study were derived from paraffin-embedded tissue blocks of colonoscopic biopsy specimens before any treatment. We used rectal endoscopic ultrasound and/or abdominopelvic CT scan to evaluate the pre-CCRT stage. The neoadjuvant CCRT was given with 5-fluorouracil-based chemotherapy and concurrent long course of radiotherapy in a daily fraction of 1.8 Gy for 25 fractions to achieve a total dose of 45Gy. After mesorectal excision, patients with $\mathrm{T} 3$ to $\mathrm{T} 4$ stage or nodal metastasis either in pre-CCRT or post-CCRT status received adjuvant systemic chemotherapy. The mean follow-up time was 48.2 months (6.2-131.2). 


\section{Histopathological assessment}

Two pathologists (HL He and CF Li) who were blinded to the patients' clinical information assessed the Histopathological findings. The staging was evaluated based on the 7th American Joint Committee on Cancer (AJCC) TNM system. We followed the criteria described by Dworak et al. to grade the degree of tumor regression after neoadjuvant CCRT [14]. Grade 0 was defined as no regression; grade 1 was defined as predominantly tumor with significant fibrosis and/or vasculopathy; grade 2 was predominantly fibrosis with scattered tumor cells; grade 3 was only scattered tumor cells in the space of fibrosis with/without acellular mucin; grade 4 was no vital tumor cells detectable.

\section{Immunohistochemical analysis and evaluation of immunostaining}

For immunohistochemical study, $3 \mu$ m-thick sections were cut from formalin-fixed,

paraffin-embedded tissue blocks of pre-CCRT biopsy specimens. After deparaffinized with xylene and rehydrated with graded ethanol, the slides were treated with $3 \%$ hydrogen peroxide for $5 \mathrm{~min}$ to quench the endogenous peroxidase activity. The slides were heated at $98^{\circ} \mathrm{C}$ of microwave, and antigen retrieval treatment was performed in a $\mathrm{pH} 6,10-\mathrm{mM}$ sodium citrate buffer for $15 \mathrm{~min}$. Then, the slides were incubated with an anti-EphA4 rabbit polyclonal primary antibody (Cat.No. ab5389, 1:100 dilution, Abcam, Cambridge, UK). Human breast carcinoma tissue was used as the positive control and normal liver tissue as the negative control. The protein expression of EphA4 was scored based on the H-score method as previously described [15-21]. The equation was as follows: H-score $=\Sigma P i(i+1)$. The " $i$ " was represented as the staining intensity $(0-3+)$ and the
" $P i$ " was the percentage of positive staining tumor cells of variable intensities $(0-100 \%)$.

\section{Statistical analysis}

All statistical analyses were carried out by the software SPSS version 14.0 (SPSS Inc., Chicago, IL, USA). The median of H-score served as a cut-off value to divide the cohort into two subgroups, low and high expression of EphA4, respectively. We used the Chi-square test $\left(X^{2}\right)$ to investigate the correlation between EphA4 expression and various clinicopathological parameters. Kaplan-Meier curves were plotted to evaluate the impact of EphA4 expression on disease-specific survival (DSS), local recurrence-free survival (LRFS) and metastasis-free survival (MeFS). The different DSS, LRFS and MeFS curves were compared using the log-rank test. Multivariate Cox proportional hazard models were performed to assess the prognostic significance of EphA4 expression and key factors correlated to three survival endpoints. A $P$-value of less than 0.05 was considered to have statistical significance.

\section{Results}

\section{EPHA4 was the top one gene significantly associated with the response to preoperative radiotherapy}

Data mining from the public transcriptome of rectal cancer revealed that there were five transmembrane receptor protein tyrosine kinase signaling pathway-associated genes, EPHA4, INSR, PTPRJ, ANGPT1, NRTN, harboring significantly different expression between the responders and non-responders to preoperative radiotherapy (Figure 1 and Table 1). With the most powerful significance $(\log 2$ ratio=1.1273 and $\mathrm{P}<0.0001)$, EphA4 was chosen for further investigation.

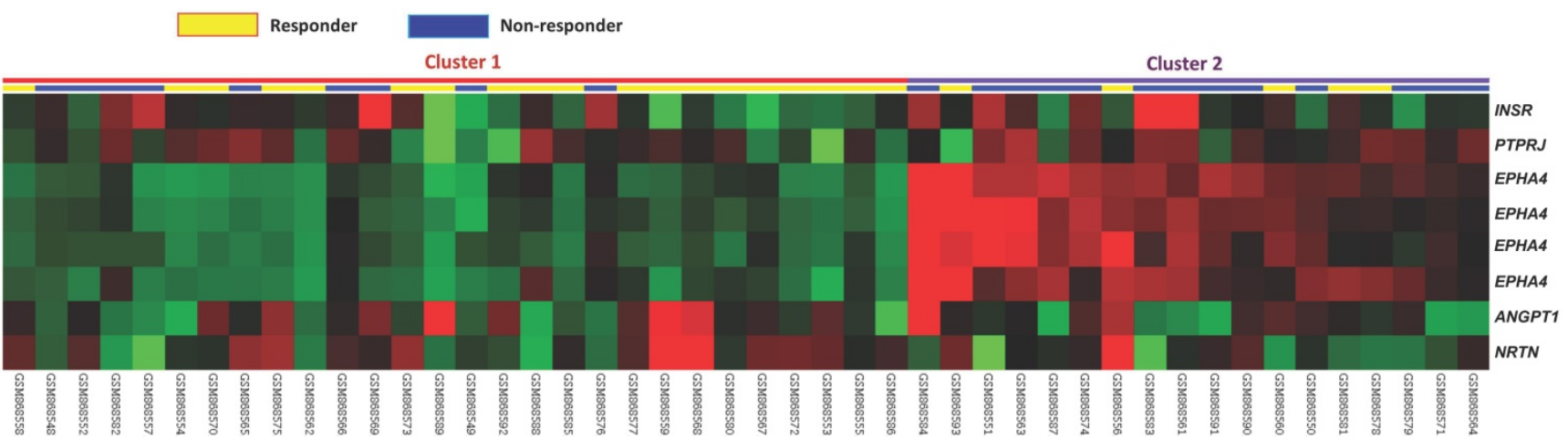

Figure 1. Analysis of gene expression profiles of rectal cancer. Analysis of microarray data from a publicly available transcriptome of rectal cancer (GSE35452) with the focus on genes associated with transmembrane tyrosine kinase receptor signaling pathway. The color-coded scale was demonstrated as red for expression levels greater than the mean and green for those lower than the mean. 
Table 1. Summary of differentially expressed genes associated with transmembrane receptor protein tyrosine kinase signaling pathway (GO:0007169) in relation to response to CCRT in rectal adenocarcinoma

\begin{tabular}{|c|c|c|c|c|c|c|}
\hline Probe & $\begin{array}{l}\text { Comparison } \\
\log 2 \text { ratio }\end{array}$ & $\begin{array}{l}\text { Comparison } \\
P \text {-value }\end{array}$ & $\begin{array}{l}\text { Gene } \\
\text { Symbol }\end{array}$ & Gene Name & Biological Process & Molecular Function \\
\hline 227449_at & 1.1273 & $<0.0001$ & EPHA4 & $\begin{array}{l}\text { EPH receptor } \\
\text { A4 }\end{array}$ & $\begin{array}{l}\text { adult walking behavior, axon guidance, } \\
\text { protein amino acid phosphorylation, } \\
\text { signal transduction, transmembrane } \\
\text { receptor protein tyrosine kinase signaling } \\
\text { pathway }\end{array}$ & $\begin{array}{l}\text { ATP binding, ephrin receptor activity, kinase } \\
\text { activity, nucleotide binding, protein kinase } \\
\text { activity, protein-tyrosine kinase activity, receptor } \\
\text { activity, transferase activity, transmembrane } \\
\text { receptor protein tyrosine kinase activity }\end{array}$ \\
\hline 228948_at & 0.7116 & $<0.0001$ & EPHA4 & $\begin{array}{l}\text { EPH receptor } \\
\text { A4 }\end{array}$ & $\begin{array}{l}\text { adult walking behavior, axon guidance, } \\
\text { protein amino acid phosphorylation, } \\
\text { signal transduction, transmembrane } \\
\text { receptor protein tyrosine kinase signaling } \\
\text { pathway }\end{array}$ & $\begin{array}{l}\text { ATP binding, ephrin receptor activity, kinase } \\
\text { activity, nucleotide binding, protein kinase } \\
\text { activity, protein-tyrosine kinase activity, receptor } \\
\text { activity, transferase activity, transmembrane } \\
\text { receptor protein tyrosine kinase activity }\end{array}$ \\
\hline 206114_at & 0.6112 & $<0.0001$ & EPHA4 & $\begin{array}{l}\text { EPH receptor } \\
\text { A4 }\end{array}$ & $\begin{array}{l}\text { adult walking behavior, axon guidance, } \\
\text { protein amino acid phosphorylation, } \\
\text { signal transduction, transmembrane } \\
\text { receptor protein tyrosine kinase signaling } \\
\text { pathway }\end{array}$ & $\begin{array}{l}\text { ATP binding, ephrin receptor activity, kinase } \\
\text { activity, nucleotide binding, protein kinase } \\
\text { activity, protein-tyrosine kinase activity, receptor } \\
\text { activity, transferase activity, transmembrane } \\
\text { receptor protein tyrosine kinase activity }\end{array}$ \\
\hline 226216_at & 0.5733 & 0.0002 & INSR & $\begin{array}{l}\text { insulin } \\
\text { receptor }\end{array}$ & $\begin{array}{l}\text { carbohydrate metabolic process, } \\
\text { generation of precursor metabolites and } \\
\text { energy, insulin receptor signaling } \\
\text { pathway, male sex determination, } \\
\text { multicellular organismal development, } \\
\text { organ morphogenesis, protein amino acid } \\
\text { autophosphorylation, protein amino acid } \\
\text { phosphorylation, protein } \\
\text { heterotetramerization, signal } \\
\text { transduction, transmembrane receptor } \\
\text { protein tyrosine kinase signaling pathway }\end{array}$ & $\begin{array}{l}\text { ATP binding, PTB domain binding, } \mathrm{SH} 2 \text { domain } \\
\text { binding, insulin binding, insulin receptor activity, } \\
\text { insulin receptor substrate binding, kinase } \\
\text { activity, nucleotide binding, phosphoinositide } \\
\text { 3-kinase binding, protein binding, protein kinase } \\
\text { activity, protein-tyrosine kinase activity, receptor } \\
\text { activity, receptor signaling protein tyrosine } \\
\text { kinase activity, signal transducer activity, } \\
\text { transferase activity, transmembrane receptor } \\
\text { protein tyrosine kinase activity, transmembrane } \\
\text { receptor protein tyrosine kinase signaling protein } \\
\text { activity }\end{array}$ \\
\hline 227396_at & 0.3956 & 0.0047 & PTPRJ & $\begin{array}{l}\text { protein } \\
\text { tyrosine } \\
\text { phosphatase; } \\
\text { receptor type; } \\
\text { J }\end{array}$ & $\begin{array}{l}\text { cell-cell signaling, dephosphorylation, } \\
\text { heart development, protein amino acid } \\
\text { dephosphorylation, transmembrane } \\
\text { receptor protein tyrosine kinase signaling } \\
\text { pathway, vasculogenesis }\end{array}$ & $\begin{array}{l}\text { hydrolase activity, phosphoprotein phosphatase } \\
\text { activity, phosphoric monoester hydrolase } \\
\text { activity, protein tyrosine phosphatase activity, } \\
\text { receptor activity, transmembrane receptor } \\
\text { protein tyrosine phosphatase activity }\end{array}$ \\
\hline 229374_at & 0.327 & 0.0035 & ЕРНA4 & $\begin{array}{l}\text { EPH receptor } \\
\text { A4 }\end{array}$ & $\begin{array}{l}\text { adult walking behavior, axon guidance, } \\
\text { protein amino acid phosphorylation, } \\
\text { signal transduction, transmembrane } \\
\text { receptor protein tyrosine kinase signaling } \\
\text { pathway }\end{array}$ & $\begin{array}{l}\text { ATP binding, ephrin receptor activity, kinase } \\
\text { activity, nucleotide binding, protein kinase } \\
\text { activity, protein-tyrosine kinase activity, receptor } \\
\text { activity, transferase activity, transmembrane } \\
\text { receptor protein tyrosine kinase activity }\end{array}$ \\
\hline 205609_at & -0.2653 & 0.0037 & ANGPT1 & angiopoietin 1 & $\begin{array}{l}\text { angiogenesis, cell differentiation, } \\
\text { multicellular organismal development, } \\
\text { signal transduction, transmembrane } \\
\text { receptor protein tyrosine kinase signaling } \\
\text { pathway }\end{array}$ & receptor binding \\
\hline 210683_at & -0.1394 & 0.0051 & NRTN & neurturin & $\begin{array}{l}\text { MAPKKK cascade, nerve development, } \\
\text { nervous system development, neural crest } \\
\text { cell migration, neurite development, } \\
\text { transmembrane receptor protein tyrosine } \\
\text { kinase signaling pathway }\end{array}$ & growth factor activity, receptor binding \\
\hline
\end{tabular}

\section{Immunohistochemical expression of EphA4 and its relationship to clinicopathological parameters}

The immunostaining EphA4 in normal mucosa, adenoma and adenocarcinoma is illustrated in Figure 2. Each sample from rectal cancer patient was assigned successfully with a $\mathrm{H}$-score (range: 105-325; median: 220). The median was regarded as a cut-off value to separated patients into two subgroups with high or low expression of EphA4, respectively. As shown in Table 2, high expression of EphA4 was significantly associated with pre-treatment (pre-Tx) lymph node metastasis (N1-2 versus N0; $P=0.004$ ), post-treatment (post-Tx) depth of tumor invasion (T3-T4 versus T1-T2; $P=0.006$ ), post-Tx lymph node metastasis (N1-2 versus N0; $P=0.011)$, vascular invasion $(P=0.015)$, and tumor regression grade $(P=0.031)$.

\section{High expression of EphA4 was associated with poor prognosis in rectal cancer patients}

At univariate analysis (Table 3 and Figure 3), we found that DSS was significantly associated with post-Tx tumor status $(P=0.0006)$, vascular invasion $(P=0.0184)$, tumor regression grade $(P=0.0038)$ and EphA4 expression $(P=0.0009)$. LRFS was significantly associated with pre-Tx nodal status $(P=0.0070)$, post-Tx tumor status $(P=0.0040)$, vascular invasion $(P=0.0028)$, and tumor regression grade $(P=0.0090)$. MeFS was significantly associated with post-Tx tumor status $(P=0.0033)$, tumor regression grade $(P=0.0006)$ 
and EphA4 expression $(P=0.0001)$. At multivariate Cox proportional-hazards regression analysis (Table 4), Tumor regression grade, as expected, remained a significant prognostic factor for DSS (HR, 2.100; 95\% CI, 1.063-4.167; $P=0.033$ ), LRFS (HR, 2.941; 95\% CI, 1.335-6.494; $P=0.007$ ) and MeFS. (HR, 2.415; 95\% CI, 1.214-4.808; $P=0.012)$. Of the most importance, EphA4 overexpression was an independent negative prognostic factor for DSS (HR, 2.528; 95\% CI, 1.131-5.651; $P=0.024)$ and MeFS (HR, 3.908; 95\% CI, $1.590-9.601 ; P=0.003$ ) after adjusting for the following clinical paramemters: tumor regression grade, vascular invasion, post-Tx tumor status and pre-Tx nodal status.
Table 2. Associations and comparisons between EphA4 expression and clinicopathological factors in 172 rectal cancer patients receiving neoadjuvant CCRT.

\begin{tabular}{|c|c|c|c|c|c|}
\hline \multirow[t]{2}{*}{ Parameter } & & \multirow[t]{2}{*}{ No. } & \multicolumn{2}{|c|}{ EphA4 Expression } & \multirow[t]{2}{*}{$P$ value } \\
\hline & & & Low Exp & High Exp. & \\
\hline \multirow[t]{2}{*}{ Gender } & Male & 108 & 52 & 56 & 0.528 \\
\hline & Female & 64 & 34 & 30 & \\
\hline \multirow[t]{2}{*}{ Age } & $<70$ & 106 & 54 & 52 & 0.754 \\
\hline & $\geqq 70$ & 66 & 32 & 34 & \\
\hline \multirow[t]{2}{*}{ Pre-Tx tumor status } & $\mathrm{T} 1-\mathrm{T} 2$ & 81 & 46 & 35 & 0.093 \\
\hline & T3-T4 & 91 & 40 & 51 & \\
\hline \multirow[t]{2}{*}{ Pre-Tx nodal status } & N0 & 125 & 71 & 54 & $0.004^{*}$ \\
\hline & N1-N2 & 47 & 15 & 32 & \\
\hline \multirow[t]{2}{*}{ Post-Tx tumor status } & $\mathrm{T} 1-\mathrm{T} 2$ & 86 & 52 & 34 & $0.006^{*}$ \\
\hline & T3-T4 & 86 & 34 & 52 & \\
\hline \multirow[t]{2}{*}{ Post-Tx nodal status } & No & 123 & 69 & 54 & $0.011^{*}$ \\
\hline & N1-N2 & 49 & 17 & 32 & \\
\hline \multirow[t]{2}{*}{ Vascular invasion } & Absent & 157 & 83 & 74 & $0.015^{*}$ \\
\hline & Present & 15 & 3 & 12 & \\
\hline \multirow[t]{2}{*}{ Perineural invasion } & Absent & 167 & 84 & 83 & 0.650 \\
\hline & Present & 5 & 2 & 3 & \\
\hline \multirow[t]{3}{*}{ Tumor regression grade } & Grade 0-1 & 37 & 14 & 23 & $0.031^{*}$ \\
\hline & Grade 2 3 & 118 & 59 & 59 & \\
\hline & Grade 4 & 17 & 13 & 4 & \\
\hline
\end{tabular}

*, statistically significant
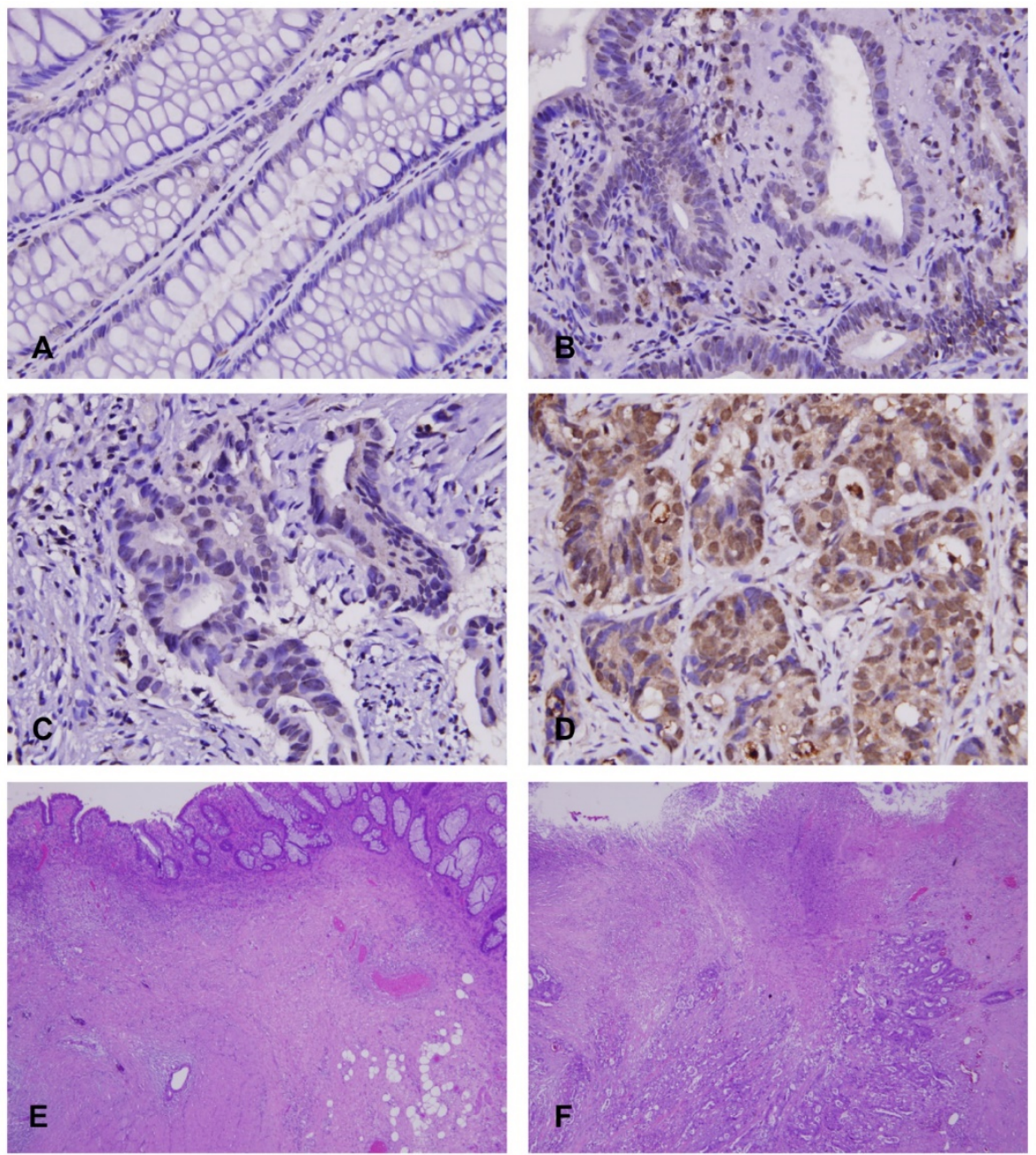

Figure 2. Immunohistochemical expression of EphA4 in rectal cancer tissues. It showed no or faint expression in normal mucosa (A) and adenoma (B), whereas variable expression was observed in adenocarcinomas: low expression $(\mathbf{C})$ and high expression $(\mathbf{D})$, which correlated with high grade $(\mathbf{E})$ and low grade $(\mathbf{F})$ of tumor regression after CCRT, respectively. 
Table 3. Univariate log-rank analysis for important clinicopathological variables and EphA4 expression

\begin{tabular}{|c|c|c|c|c|c|c|c|c|}
\hline \multirow[t]{2}{*}{ Parameter } & & \multirow[b]{2}{*}{ No. of case } & \multicolumn{2}{|l|}{ DSS } & \multicolumn{2}{|l|}{ LRFS } & \multicolumn{2}{|l|}{ MeFS } \\
\hline & & & No. of event & $P$ value & No. of event & $P$ value & No. of event & $P$ value \\
\hline \multirow[t]{2}{*}{ Gender } & Male & 108 & 20 & 0.9026 & 7 & 0.2250 & 17 & 0.3520 \\
\hline & Female & 64 & 11 & & 20 & & 14 & \\
\hline \multirow[t]{2}{*}{ Age } & $<70$ & 106 & 19 & 0.8540 & 18 & 0.6615 & 20 & 0.7427 \\
\hline & $\geqq 70$ & 66 & 12 & & 9 & & 11 & \\
\hline \multirow[t]{2}{*}{ Pre-Tx tumor status } & T1-T2 & 81 & 10 & 0.0776 & 10 & 0.2261 & 11 & 0.1745 \\
\hline & T3-T4 & 91 & 21 & & 17 & & 20 & \\
\hline \multirow[t]{2}{*}{ Pre-Tx nodal status } & N0 & 125 & 19 & 0.0711 & 15 & $0.0070^{*}$ & 19 & 0.0973 \\
\hline & N1-N2 & 47 & 21 & & 12 & & 12 & \\
\hline \multirow[t]{2}{*}{ Post-Tx tumor status } & $\mathrm{T} 1-\mathrm{T} 2$ & 86 & 7 & $0.0006^{*}$ & 7 & $0.0040^{*}$ & 8 & $0.0033^{*}$ \\
\hline & T3-T4 & 86 & 24 & & 20 & & 23 & \\
\hline \multirow[t]{2}{*}{ Post-Tx nodal status } & N0 & 123 & 21 & 0.5998 & 16 & 0.1320 & 20 & 0.4634 \\
\hline & N1-N2 & 49 & 10 & & 11 & & 11 & \\
\hline \multirow[t]{2}{*}{ Vascular invasion } & Absent & 157 & 25 & $0.0184^{*}$ & 21 & $0.0028^{*}$ & 27 & 0.4470 \\
\hline & Present & 15 & 6 & & 6 & & 4 & \\
\hline \multirow[t]{2}{*}{ Perineural invasion } & Absent & 167 & 29 & 0.2559 & 25 & 0.0940 & 30 & 0.9083 \\
\hline & Present & 5 & 2 & & 2 & & 1 & \\
\hline \multirow[t]{3}{*}{ Tumor regression grade } & Grade $0-1$ & 37 & 13 & $0.0038^{*}$ & 10 & $0.0090^{*}$ & 14 & $0.0006^{*}$ \\
\hline & Grade 2 3 & 118 & 17 & & 17 & & 16 & \\
\hline & Grade 4 & 17 & 1 & & 0 & & 1 & \\
\hline \multirow[t]{2}{*}{ EphA4 expression } & Low Exp. & 86 & 9 & $0.0009^{*}$ & 11 & 0.0719 & 6 & $0.0001^{*}$ \\
\hline & High Exp. & 86 & 22 & & 16 & & 25 & \\
\hline
\end{tabular}

DSS, disease-specific survival; LRFS, local recurrence-free survival; MeFS, metastasis-free survival; *, statistically significant

Table 4. Multivariate analysis

\begin{tabular}{|c|c|c|c|c|c|c|c|c|c|}
\hline \multirow[t]{2}{*}{ Parameter } & \multicolumn{3}{|l|}{ DSS } & \multicolumn{3}{|l|}{ LRFS } & \multicolumn{3}{|l|}{ MeFS } \\
\hline & H.R. & $95 \% \mathrm{CI}$ & $P$ Value & H.R. & $95 \% \mathrm{CI}$ & $P$ Value & H.R. & $95 \% \mathrm{CI}$ & $P$ Value \\
\hline $\begin{array}{l}\text { Tumor regression } \\
\text { grade }\end{array}$ & 2.100 & $1.063-4.167$ & $0.033^{*}$ & 2.941 & $1.335-6.494$ & $0.007^{*}$ & 2.415 & $1.214-4.808$ & $0.012^{*}$ \\
\hline EphA4 expression & 2.528 & $1.131-5.651$ & $0.024^{*}$ & - & - & - & 3.908 & $1.590-9.601$ & $0.003^{*}$ \\
\hline Vascular invasion & 2.017 & $0.795-5.120$ & 0.140 & 3.034 & $1.093-8.420$ & $0.033^{*}$ & - & - & - \\
\hline Post-Tx tumor status & 2.514 & $1.051-6.051$ & $0.038^{*}$ & 2.141 & $0.863-5.311$ & 0.101 & 1.910 & $0.831-4.387$ & 0.127 \\
\hline Pre-Tx nodal status & - & - & - & 1.849 & $0.969-3.527$ & 0.062 & - & - & - \\
\hline
\end{tabular}

H.R., hazard ratio; CI, confidence interval; DSS, disease-specific survival; LRFS, local recurrence-free survival; MeFS, metastasis-free survival; *, statistically significant.
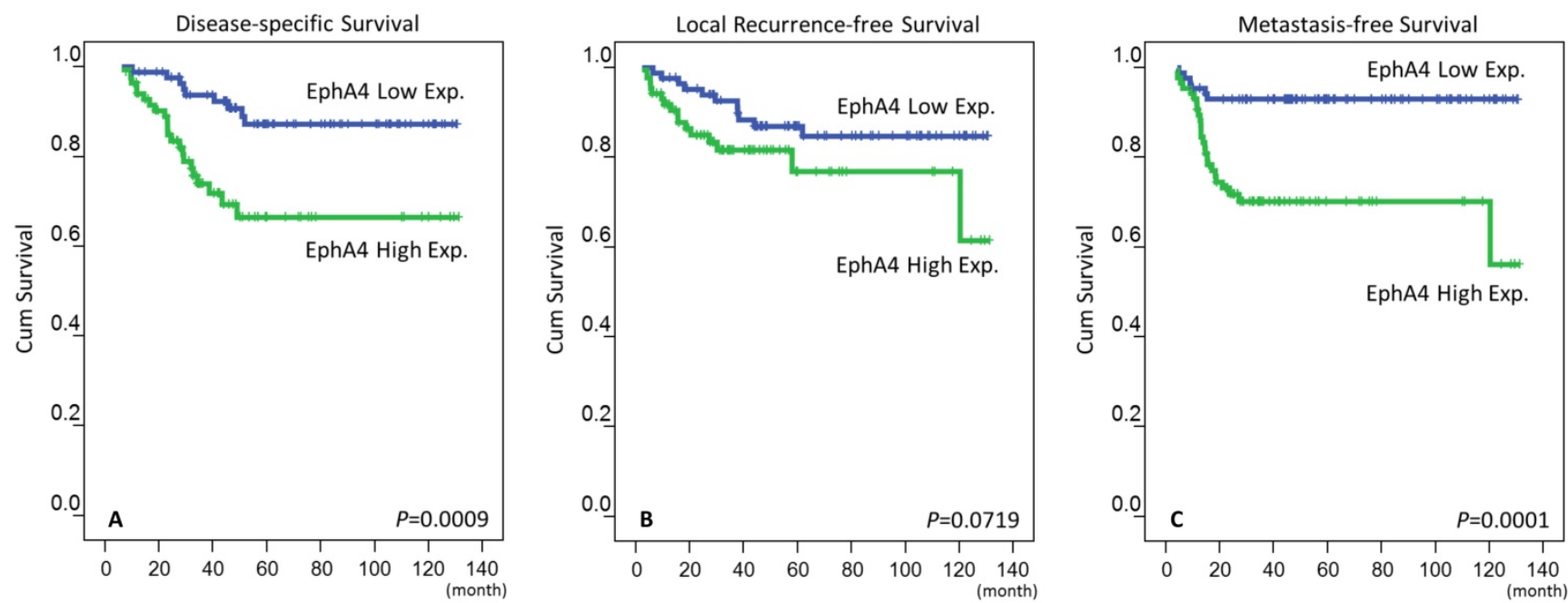

Figure 3. Survival of patients. Kaplan-Meier survival curves of disease-specific survival (A), local recurrence-free survival (B) and metastasis-free survival (C) in patients with rectal cancer according to the expression of EphA4.

\section{Discussion}

Our result suggested that high expression EphA4 was associated with advanced disease status, poor response to preoperative CCRT and worse outcomes. EphA4 was an independent prognostic factor in rectal cancer, particularly in disease-related death and distant metastasis. In gastric cancer, overexpression of EphA4 has been shown to significantly correlate with depth of tumor invasion, 
recurrence, and shorter overall survival. In In vitro study, suppression of EphA4 expression by siRNA in EphA4-overexpressing gastric cancer cell lines significantly inhibited cell growth [11]. In pancreatic cancer cells, the author demonstrated similar finding that knocking down EphA4 expression drastically attenuated the viability of pancreatic ductal adenocarcinoma cells [12]. In 2008, overexpression of EphA4 gene has been found to be associated with liver metastasis in colorectal cancer. In that study, however, there was no correlation between EphA4 expression and other clinicopathological factors, such as depth of invasion, nodal metastasis or lymphovascular invasion [10]. In our study, we found high expression of EphA4 was significantly associated with advanced pre-Tx nodal status and increased vascular invasion, indicating an aggressive phenotype. More importantly, after neoadjuvant treatment, it significantly correlated with advanced post-Tx tumor status, post-Tx nodal status and lesser degree of tumor regression. Accordingly, EphA4 was an important predictive factor for CCRT response.

In the human genome, there are 9 EphA and 5 EphB receptors that bind to glycosylphosphatidylinositol (GPI)-linked ephrin-A ligands and transmembrane ephrin- $B$ ligands, respectively [22]. EphA4 is the exception that possesses the ability to bind to both ephrin-A and ephrin-B ligands [23]. These Eph receptor tyrosine kinases contribute to contact-dependent bidirectional signaling into neighboring cells through interacting with membrane-bound ephrin family ligands residing on adjacent cells. The forward signaling, defined as signaling pathway downstream of the receptors, depends on Eph kinase activity propagating in the receptor-expressing cells. In the opposite way, the signaling pathway downstream of the ephrin ligands is referred to as reverse signaling, depending on Src family kinase propagating in the ephrin-expressing cells [24-26]. Upon activation by ephrin ligands, Eph signaling can modulate cell morphology, integrin-dependent cell adhesion, migration and invasion by regulation of the Rac, Rap and Rho GTPases activity and modifying the organization of the cytoskeleton [22, 27]. Numerous Eph receptors and ephrins are not only upregulated in cancer tissues but also in a subset of tumor microenvironment, such as endothelial cells [28, 29]. However, the expression and regulatory role of Eph and ephrin expression in other tumor compartments, such as activated fibroblasts and infiltrating inflammatory cells is poorly understood.

In human glioma U251 cell line, EphA4 was found to form a heteroreceptor complex with FGFR1 and that the EphA4/ FGFR1 complex enhanced cell migration and proliferation through promoting FGFR-mediated downstream signal transduction [13]. Moreover, a recent study demonstrated that EphA4 was responsible for regulating the aggressive phenotype of irradiated colorectal cancer cells [30]. Irradiation could increase the activation level of the survivor progeny of colorectal cancer cells, and thus promote the internalization of a complex E-cadherin-EphA4, leading to cell-cell adhesion disruption. In addition, knockdown of EphA4 decreased the invasive and migratory abilities, as well as metalloprotease activity induced by irradiation. These biological behaviors were mediated by AKT and ERK1/2 signaling, and the ERK1/2 activity was affected by EphA4. In line with this, our data supported this finding that high expression of EphA4 was significantly associated with a radioresistant phenotype characterized by lesser degree of tumor regression after CCRT.

The activation of Eph signaling depends on direct interaction between cells that harboring Eph receptors and ephrin ligands. Blockage of these receptor-ligand interactions can reduce the effect of Eph on tumor progression. Some small peptides, such as KYL, APY, and VTM peptides have been found to antagonize ephrin binding and EphA4 activation in a competitive manner [31]. Furthermore, through a high throughput screening to find small molecule inhibitors of EphA4 for targeted therapy, a small molecule, 2,5-dimethylpyrrolyl benzoic acid derivative, was identified to be able to inhibit binding of natural ephrin ligands or peptide ligands to the extracellular domain of the EphA4 receptor [32]. Though the therapeutic effect and adverse reaction of these agents on human is still unknown, it indeed sheds light on providing targeted therapy for patients with EphA4-high expressing cancers.

In conclusion, our study suggested that EphA4 overexpression was significantly associated with tumor progression. More importantly, high expression of EphA4 predicted poorer response to neoadjuvant CCRT. EphA4 was a promising and independent prognostic factor in rectal cancer. EphA4 may act as a potential target for personalized therapy in patients with rectal cancer treated with neoadjuvant CCRT combined with total mesorectal excision.

\section{Abbreviations}

CCRT: concurrent chemoradiotherapy; EphA4: Eph receptors A4; EUS: endoscopic ultrasound; Pre-Tx: pre-treatment; Post-Tx: post-treatment; AJCC: American Joint Committee on Cancer; DSS: disease-specific survival; LRFS: local recurrence-free survival; MeFS: metastasis-free survival 


\section{Acknowledgements}

This study is supported by Chi-Mei Medical Center (CLFHR10534). This study was also supported by grants from Kaohsiung Medical University "Aim for the Top Universities" (KMU-TP104E31, KMU-TP104G00, KMU-TP104G01, KMU-TP104G04), the health and welfare surcharge of tobacco products, Ministry of Health and Welfare (MOHW105-TDU-B-212-134007) and E-Da Hospital (EDAHP105012). The authors thank the BioBank of Chi Mei Medical Center for providing the tumor samples.

\section{Competing Interests}

The authors have declared that no competing interest exists.

\section{References}

1. Cancer leading cause of death in Taiwan. http://www.mohw.gov.tw/cht/Ministry/DM2_P.aspx?f_list_no=7\&fod_list no $=5313 \&$ doc no $=49778$. Accessed $26 \mathrm{Jul} 2015$.

2. Read TE, McNevin MS, Gross EK, Whiteford HM, Lewis JL, Ratkin G, et al. Neoadjuvant therapy for adenocarcinoma of the rectum: tumor response and acute toxicity. Dis Colon Rectum. 2001; 44: 513-22.

3. Gerard JP, Conroy T, Bonnetain F, Bouche O, Chapet O, Closon-Dejardin MT, et al. Preoperative radiotherapy with or without concurrent fluorouracil and leucovorin in T3-4 rectal cancers: results of FFCD 9203. J Clin Oncol. 2006; 24: 4620-5.

4. Bosset JF, Collette L, Calais G, Mineur L, Maingon P, Radosevic-Jelic L, et al. Chemotherapy with preoperative radiotherapy in rectal cancer. N Engl J Med. 2006; 355: 1114-23

5. Watanabe T, Komuro Y, Kiyomatsu T, Kanazawa T, Kazama Y, Tanaka J, et al. Prediction of sensitivity of rectal cancer cells in response to preoperative radiotherapy by DNA microarray analysis of gene expression profiles. Cancer Res. 2006; 66: 3370-4.

6. Unified nomenclature for Eph family receptors and their ligands, the ephrins. Eph Nomenclature Committee. Cell. 1997; 90: 403-4.

7. Pasquale EB. Eph receptors and ephrins in cancer: bidirectional signalling and beyond. Nat Rev Cancer. 2010; 10: 165-80.

8. Brantley-Sieders DM, Chen J. Eph receptor tyrosine kinases in angiogenesis: from development to disease. Angiogenesis. 2004; 7: 17-28.

9. Brantley-Sieders D, Schmidt S, Parker M, Chen J. Eph receptor tyrosine kinases in tumor and tumor microenvironment. Curr Pharm Des. 2004; 10: 3431-42.

10. Oshima. Overexpression of EphA4 gene and reduced expression of EphB2 gene correlates with liver metastasis in colorectal cancer. International Journal of Oncology. 1992

11. Oki M. Overexpression of the receptor tyrosine kinase EphA4 in human gastric cancers. World Journal of Gastroenterology. 2008; 14: 5650

12. Iiizumi M, Hosokawa M, Takehara A, Chung S, Nakamura T, Katagiri T, et al. EphA4 receptor, overexpressed in pancreatic ductal adenocarcinoma, promotes cancer cell growth. Cancer Sci. 2006; 97: 1211-6.

13. Fukai I, Yokote $H$, Yamanaka $R$, Arao $T$, Nishio $K$, Itakura $T$. EphA4 promotes cell proliferation and migration through a novel EphA4-FGFR1 signaling pathway in the human glioma U251 cell line. Mol Cancer Ther. 2008; 7: 2768-78

14. Dworak O, Keilholz L, Hoffmann A. Pathological features of rectal cancer after preoperative radiochemotherapy. Int J Colorectal Dis. 1997; 12: 19-23.

15. Wu YT, Yen SL, Li CF, Chan TC, Chen TJ, Lee SW, et al. Overexpression of Transient Receptor Protein Cation Channel Subfamily A Member 1, Confers an Independent Prognostic Indicator in Nasopharyngeal Carcinoma. J Cancer. 2016; 7: 1181-8

16. Liao KM, Chao TB, Tian YF, Lin CY, Lee SW, Chuang HY, et al. Overexpression of the PSAT1 Gene in Nasopharyngeal Carcinoma Is an Indicator of Poor Prognosis. J Cancer. 2016; 7: 1088-94.

17. Lee $\mathrm{YY}, \mathrm{Wu} \mathrm{WJ}$, Huang $\mathrm{CN}, \mathrm{Li} \mathrm{CC}, \mathrm{Li} \mathrm{WM}$, Yeh BW, et al. CSF2 Overexpression Is Associated with STAT5 Phosphorylation and Poor Prognosis in Patients with Urothelial Carcinoma. J Cancer. 2016; 7: 711-21.

18. He HL, Lee YE, Liang PI, Lee SW, Chen TJ, Chan TC, et al. Overexpression of JAK2: a predictor of unfavorable prognosis for nasopharyngeal carcinoma. Future Oncol. 2016; 12: 1887-96.

19. Chao TB, Li CF, Lin CY, Tian YF, Chang IW, Sheu MJ, et al. Prognostic significance of DSG3 in rectal adenocarcinoma treated with preoperative chemoradiotherapy. Future Oncol. 2016; 12: 1457-67.
20. Chang IW, Lin VC, Wu WJ, Liang PI, Li WM, Yeh BW, et al. Complement Component 1, s Subcomponent Overexpression is an Independent Poor Prognostic Indicator in Patients with Urothelial Carcinomas of the Upper Urinary Tract and Urinary Bladder. J Cancer. 2016; 7: 1396-405.

21. Chang IW, Li CF, Lin VC, He HL, Liang PI, Wu WJ, et al. Prognostic Impact of Thrombospodin-2 (THBS2) Overexpression on Patients with Urothelial Carcinomas of Upper Urinary Tracts and Bladders. J Cancer. 2016; 7: 1541-9.

22. Pasquale EB. Eph receptor signalling casts a wide net on cell behaviour. Nat Rev Mol Cell Biol. 2005; 6: 462-75.

23. Gale NW, Holland SJ, Valenzuela DM, Flenniken A, Pan L, Ryan TE, et al. Eph receptors and ligands comprise two major specificity subclasses and are reciprocally compartmentalized during embryogenesis. Neuron. 1996; 17: 9-19.

24. Miao H, Strebhardt K, Pasquale EB, Shen TL, Guan JL, Wang B. Inhibition of integrin-mediated cell adhesion but not directional cell migration requires catalytic activity of EphB3 receptor tyrosine kinase. Role of Rho family small GTPases. J Biol Chem. 2005; 280: 923-32.

25. Matsuoka H, Obama H, Kelly ML, Matsui T, Nakamoto M. Biphasic functions of the kinase-defective Ephb6 receptor in cell adhesion and migration. J Biol Chem. 2005; 280: 29355-63.

26. Gu C, Park S. The EphA8 receptor regulates integrin activity through p110gamma phosphatidylinositol-3 kinase in a tyrosine kinase activity-independent manner. Mol Cell Biol. 2001; 21: 4579-97.

27. Pasquale EB. Eph-ephrin bidirectional signaling in physiology and disease. Cell. 2008; 133: 38-52.

28. Pandey A, Shao H, Marks RM, Polverini PJ, Dixit VM. Role of B61, the ligand for the Eck receptor tyrosine kinase, in TNF-alpha-induced angiogenesis. Science. 1995; 268: 567-9.

29. Cheng N, Brantley DM, Liu H, Lin Q, Enriquez M, Gale N, et al. Blockade of EphA receptor tyrosine kinase activation inhibits vascular endothelial cell growth factor-induced angiogenesis. Mol Cancer Res. 2002; 1: 2-11.

30. de Marcondes PG, Bastos LG, de-Freitas-Junior JC, Rocha MR, Morgado-Diaz JA. EphA4-mediated signaling regulates the aggressive phenotype of irradiation survivor colorectal cancer cells. Tumour Biol. 2016.

31. Murai KK, Nguyen LN, Koolpe M, McLennan R, Krull CE, Pasquale EB. Targeting the EphA4 receptor in the nervous system with biologically active peptides. Mol Cell Neurosci. 2003; 24: 1000-11.

32. Noberini R, Koolpe M, Peddibhotla S, Dahl R, Su Y, Cosford ND, et al. Small molecules can selectively inhibit ephrin binding to the EphA4 and EphA2 receptors. J Biol Chem. 2008; 283: 29461-72. 